\title{
In Situ Bragg Coherent Diffraction Imaging Study of a Cement Phase Microcrystal during Hydration
}

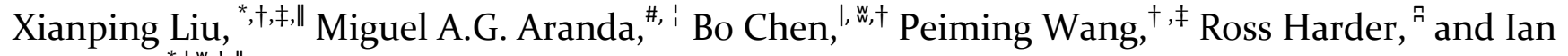 \\ Robinson ${ }^{*}$, ,w, $\dagger, \|$ \\ ${ }^{\dagger}$ School of Materials Science and Engineering, Tongji University, Shanghai 201804, China \\ ${ }^{\ddagger}$ Key Laboratory of Advanced Civil Engineering Materials (Tongji University), Ministry of Education, Tongji Univer- \\ sity, Shanghai 201804, China \\ \#Departamento de Química Inorgánica, Cristalografía y Mineralogía, Universidad de Málaga, 29071-Málaga, Spain \\ 'ALBA Synchrotron, Carretera BP 1413, Km. 3.3, E-0829o Cerdanyola, Barcelona, Spain \\ 'London Centre for Nanotechnology, University College London, London $\mathrm{WC}_{1} \mathrm{H}$ oAH, UK \\ "Research Complex at Harwell, Rutherford Appleton Laboratory, Oxfordshire OXı1 oFA, UK \\ ${ }^{\text {F}}$ Advanced Photon Source, Argonne National Laboratory, Argonne IL 60439, USA
}

\section{Supporting Information}

\begin{abstract}
Results of Bragg coherent diffraction imaging (BCDI) confirm the ion migration and consumption during hydration of calcium monoaluminate (CA). The chemical phase transformation promotes the hydration process and the formation of new hydrates. There is a potential for the formation of hydrates near where the active ions accumulate. BCDI has been used to study the in situ hydration process of CA over a 3 day period. The evolution of three-dimensional (3D) Bragg diffraction electron density (Bragg density) and strain fields present on the nano-scale within the crystal were measured and visualized. Initial Bragg densities and strains in CA crystal derived from sintering evolve into various degrees during hydration. The variation of Bragg density within the crystal is attributed to the change of the degree of crystal ordering, which could occur through ion transfer during hydration. The observed strain, coming from interfacial mismatch effect between high Bragg density and low Bragg density parts in the crystal, remained throughout the experiment. The first Bragg density change during hydration process is due to a big loss of Bragg density as seen in the image amplitude but not its phase. This work provides new evidence supporting the through-solution reaction mechanism of CA.
\end{abstract}

Calcium monoaluminate, $\mathrm{CaAl}_{2} \mathrm{O}_{4}(\mathrm{CA})$ in cement nomenclature, is the main active mineralogical component of calcium aluminate cements (CACs). These cements are mainly used in the applications at high temperature, such as refractory bricks, and also for winter construction works. ${ }^{1}$ CA crystallizes in a monoclinic crystal structure through solid state reaction between $\mathrm{CaO}$ and $\mathrm{Al}_{2} \mathrm{O}_{3}{ }^{2.3}$ by sintering. This cement constituent was selected for study because of its fast hydration kinetics (faster than the main constituent of Portland cements, tricalcium silicate, $\mathrm{C}_{3} \mathrm{~S}$ ). CA can be prepared with clean flat facets, and it can serve as a model for cement hydration.

The hydration mechanism of CA is well known as throughsolution reaction using various analysis technologies. ${ }^{4,5}, 6$ The CA hydration process arises by dissolution in water of reactants and the achievement of saturated solution. Subsequently, the crystallization of calcium aluminate hydrates occurs, as a final process, the hydrate crystallization is highly temperature dependent. ${ }^{7}$ At temperatures below ${ }^{10} \mathrm{C}$, the formation of hexagonal $\mathrm{CAH}_{10}\left(\mathrm{C}=\mathrm{CaO}, \mathrm{A}=\mathrm{Al}_{2} \mathrm{O}_{3}, \mathrm{H}=\right.$ $\mathrm{H}_{2} \mathrm{O}$ ) is favored by reaction [1].

$$
\mathrm{CA}+10 \mathrm{H} \rightarrow \mathrm{CAH}_{10}
$$

Once above $10^{\circ} \mathrm{C}$, the formation of another hexagonal calcium aluminate hydrate ${ }^{8}\left(\mathrm{C}_{2} \mathrm{AH}_{8}\right)$ with $\mathrm{AH}_{3}$ may occur through the reaction [2], jointly with the reaction [1]. $\mathrm{AH}_{3}$ is initially an amorphous hydrated aluminum oxide gel that later may crystallize as hexagonal gibbsite.

$$
2 \mathrm{CA}+11 \mathrm{H} \rightarrow \mathrm{C}_{2} \mathrm{AH}_{8}+\mathrm{AH}_{3}
$$

These hydrates $\left(\mathrm{CAH}_{10}\right.$ and $\left.\mathrm{C}_{2} \mathrm{AH}_{8}\right)$ are metastable and tend to convert to the thermodynamically stable calcium aluminate hydrate ${ }^{9}, \mathrm{C}_{3} \mathrm{AH}_{6}$, which has a hydrogarnet structure type, i.e. cubic structure, according to reactions [3] and [4].

$$
\begin{aligned}
& { }_{3} \mathrm{CAH}_{10} \rightarrow \mathrm{C}_{3} \mathrm{AH}_{6}+2 \mathrm{AH}_{3}+18 \mathrm{H} \\
& { }_{3} \mathrm{C}_{2} \mathrm{AH}_{8} \rightarrow{ }_{2} \mathrm{C}_{3} \mathrm{AH}_{6}+\mathrm{AH}_{3}+9 \mathrm{H}
\end{aligned}
$$

At higher temperatures, above $70^{\circ} \mathrm{C}$, the formation of $\mathrm{C}_{3} \mathrm{AH}_{6}$ and $\mathrm{AH}_{3}$ is following the reaction [5]. ${ }^{10}$ 


$$
{ }_{3} \mathrm{CA}+12 \mathrm{H} \rightarrow \mathrm{C}_{3} \mathrm{AH}_{6}+2 \mathrm{AH}_{3}
$$

However, due to properties of the highly temperature dependent hydration of $\mathrm{CA}$, the hydration process may also comprise chemical reactions with different hydration mechanisms. For example, at temperatures ranging from $60^{\circ} \mathrm{C}$ to $90^{\circ} \mathrm{C}$, solid-state reaction mechanism was reported using synchrotron energy-dispersive diffraction resulting layers of hydrates. ${ }^{11}$ Similar observations were found at other temperatures. $^{12,13}$

There are always having disputes between the throughsolution reaction mechanism and the solid-state reaction mechanism because it is difficult to give a definite explanation to some phenomena with a single mechanism due to the limitation of the available characterization methods. According to the through-solution reaction mechanism, due to the enormous and fast early dissolution of CA, a compact layer of hydrates formed surrounding unreacted CA crystals, precipitating in air-filled porosity homogenously and not far away from the surface of CA crystals. $\mathrm{CaO}$ has a higher solubility than $\mathrm{Al}_{2} \mathrm{O}_{3}$, therefore the surface of CA became rich in $\mathrm{Al}_{2} \mathrm{O}_{3}$ after 20 minutes of hydration. ${ }^{14}$ The composition of pore solution also changed by precipitation of non-crystalline hydrates. ${ }^{9}$ However, according to solid-state reaction mechanism, an initial film barrier of the hydrates was formed on the surface of unhydrated crystals, which prevents water molecules from reaching the unreacted CA surface. ${ }^{15,16}$ Both hydration mechanisms lead to the slowly decreased hydration rate, after the main reaction period, due to a diffusion controlled process. The diffusion species in the former mechanism is the dissolved ions from CA, and for the latter mechanism is the water. ${ }^{9,11}$ Further hydration of CA was hindered after fast early hydration. ${ }^{12,17}$

The understanding of the hydration mechanism of CA will help to establish a reliable hydration mechanism of the more complicated calcium aluminate cement systems. Hence it is the central issue about the application of cements to achieve higher level of performance. Although a great deal of the hydration mechanism of CA is already understood; however they have been confined by either the static or indirect instinct of the characterization methods, which is not comprehensive under the current technical conditions.

The structural origin of reactivity of cement clinker can be addressed by visualizing the crystal structure evolution of its minerals during hydration. Transmission electron microscope (TEM) can be used to investigate defects at the resolution of near-atomic scale or be used to characterize defect dynamics in real time. ${ }^{18}$ However the requirements for electron transparent samples make the samples themselves are usually not representative of the bulk material. In addition, the required vacuum is not compatible with hydrating conditions. Alternatively, the X-ray method to probe materials with $3 \mathrm{D}$ images ${ }^{19,20}$ or crystal structures has improved to achieve spatial resolutions of approaching $10 \mathrm{~nm} .^{21}$ Bragg Coherent Diffraction Imaging (BCDI) is such a powerful new method not only to obtain three-dimensional (3D) images of individual crystals on nano-scale through inversion of the diffraction data by computational method, but also highly sensitive to crystal defects and strain fields inside crystals seen as phase evolution. ${ }^{21-24}$ The phase measures the projection of the lattice distortion onto the direction of the meas- ured Bragg peak. Additionally, the experimental conditions are compatible with in-situ cement hydration characterization.

In this communication, we concentrate on the application of $\mathrm{BCDI}$ for investigating in situ $3 \mathrm{D}$ Bragg diffraction electron density (Bragg density) and strain evolution of a CA microcrystal during 3 days of hydration. Bragg diffraction patterns were used to select the individual CA crystals and characterize the Bragg density evolution of the crystalline parts of it. This is not the same thing as physical density because it includes all the effects of using a Bragg peak to measure it, such as crystal ordering, defects, disorder etc. Strain field distribution and evolution during hydration were recorded accordingly. We expected to find new evidence for the CA hydration mechanism.

CA was prepared by high temperature synthesis using the appropriate stoichiometric mixtures of $\mathrm{CaCO}_{3}$ and $\mathrm{Al}_{2} \mathrm{O}_{3}$, with grinding process taking place between each heating step (see the supporting information $\mathrm{S} 1$ ). The sample was measured with scanning electron microscope (SEM) and laboratory $x$-ray powder diffractometer (XRPD). The size of CA crystals was about $1 \mu \mathrm{m}$ (Figure Sia). The synthesized sample was confirmed to contain $94.9(1) \mathrm{wt} \%$ of monoclinic CA (see the supporting information $\mathrm{S}_{2}$ ). Some of the CA was ground into microcrystals and spread on a clean silicon wafer substrate, and then heated in an oven at $500{ }^{\circ} \mathrm{C}$ for half an hour to be attached on the silicon wafer for coherent diffraction data collection. Then the silicon wafer together with the sample was wrapped with Kapton films to form an open chamber. After the data collection of a selected unhydrated CA crystal, saturated potassium sulphate solution was injected into a container inside the chamber. The chamber was then sealed with the sticky side of the Kapton film to get a relative humidity of $98 \%$ at $20^{\circ} \mathrm{C}$ inside the Kapton bag (Figure 1 ). The CA microcrystal began to hydrate when it contacted with the water vapour, and the data collections of the same CA crystal as that before hydration were performed subsequently at different time intervals. After the experiment was finished, the silicon wafers, containing partially hydrated crystals, were removed from the bag, sealed and later studied by SEM.

The BCDI measurement was made at the beamline 34-ID$C$ at the Advanced Photon Source (APS). The schematic experimental setup is illustrated in Figure 1. Samples were mounted on kinematic mounts with a reproducibility position accuracy of 1-2 microns. With the cooperation of a confocal microscope, the same crystal can be measured repeatedly over a long period. The coherent monochromatic X-ray beam ( $9 \mathrm{keV})$ was defined by passing through an entrance slit of $60(\mathrm{v}) \times 30(\mathrm{~h})$ microns, then focused by the Kirkpatrick-Baez (KB) mirrors to a size around 1 micron. Initially, the diffraction peaks were located by a $2 \mathrm{D}$ Pilatus detector, then the diffraction from the sample was collected by a ChargeCoupled Device (CCD) detector located $1 \mathrm{~m}$ away. $3 \mathrm{D}$ data were acquired as stacks of $2 \mathrm{D}$ CCD frames collected over rocking curves ${ }^{16}$ of the principle sample rotation axis, perpendicular to the X-ray beam. A typical scan spans over 0.4 degree range (which may slightly depends upon the width of the recorded signal for the reflection) with a rocking angle of $0.005^{\circ}$ which resulted in 80 frames. Each frame was recorded for 2 seconds, and to improve the statistics 15 acquisitions 
were taken. Therefore, it took 40 minutes to collect one full set of $3 \mathrm{D}$ data.

These $3 \mathrm{D}$ diffraction patterns were inverted to $3 \mathrm{D}$ images using Fienup's Hybrid Input-Output algorithm ${ }^{25}$ to phase retrieve the diffraction patterns ${ }^{24}$. The "support" function describing the crystal shape was derived from using a "shrinkwrap" procedure ${ }^{26}$ from the initial data set and kept fixed for the time-evolved images. The final images were transformed to Cartesian laboratory coordinates for viewing $^{24}$. Results showing isosurface view (Figure 2) and crosssectional views (Figure 3 and Figure 4) of the imaged crystal, which visualize the outer surface and the interior of the crystal, are presented in this report. The spatial resolution of the resulting $3 \mathrm{D}$ images is around $30 \mathrm{~nm} .{ }^{16}$ The densities of the CA crystal were detected by the amplitude of this Bragg density function (Figure 3), which was interpreted as the degree of crystal ordering coupled by the Bragg peak. Strains were detected as phase in the real-space images (Figure 4), which was interpreted as the projection of a $3 \mathrm{D}$ deformation field arising from sintering, interfacial contact forces between the crystals, interfacial mismatching effect between high Bragg density and low Bragg density parts, as well as from the dissolution force of etch pit on the crystal surface.

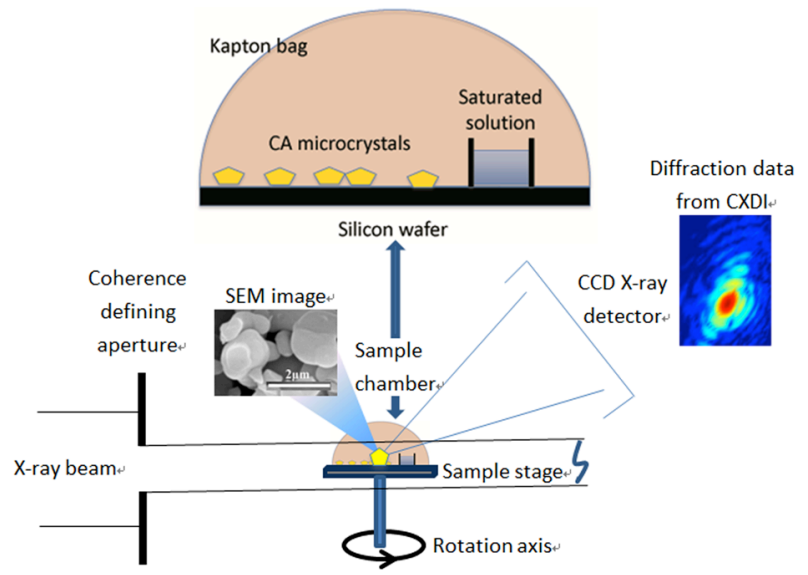

Figure 1. Schematic sample chamber and experiment setup at the beamline 34-ID-C at APS.

Figure 2 shows translucent isosurface view of the reconstructed $3 \mathrm{D}$ image of the CA crystal during hydration with two of its cut-through sections rendered in colour. The reconstructed $3 \mathrm{D}$ images of the crystal with time evolution in Figure 3 and Figure 4 are shown in two sections since they can well represent the crystal as Figure 2 shows.

Figure 3 shows the two cross sections ( $\mathrm{z}=5$ and $\mathrm{z}=9$ ) through the reconstructed $3 \mathrm{D}$ images of the Bragg density of a single CA microcrystal during 3 days of hydration. The Bragg density is coded in colour from dark blue to red (from o to 1 ), all the images are on the same scale. The Bragg density values are all relative within each image. However, the maximum does not vary much from one time to the next. Higher values of Bragg density correspond to higher degrees of crystal ordering. In most cases, the high Bragg density areas are surrounded with gradually lower Bragg density areas in the crystal, which indicates there is a high crystal ordering centre in the crystal. The periphery of the cross section is of the lowest Bragg density since the crystal dissolution starts from the surface.

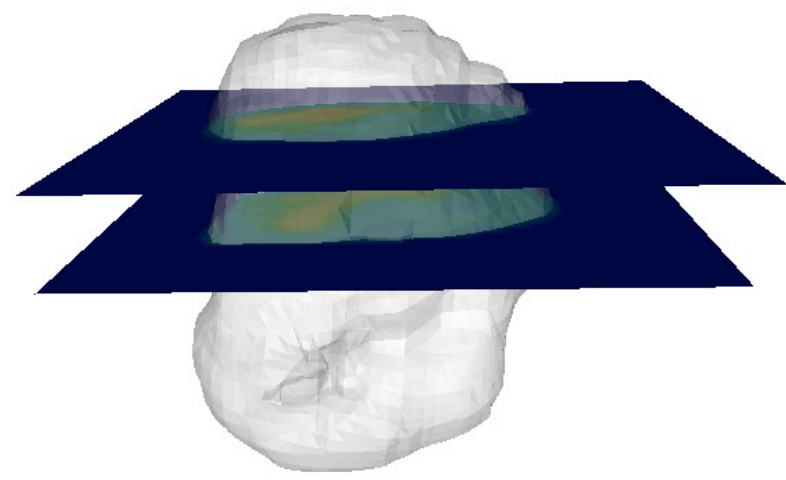

Figure 2. 50\% translucent isosurface view of the outer surface of the imaged crystal showing the slice that was measured during its hydration.

It can be hypothesised, that if the hydration is controlled by the through-solution reaction mechanism, the $\mathrm{CaO}$ and $\mathrm{Al}_{2} \mathrm{O}_{3}$ ions originally present in the CA crystalline lattice diffuse from the interior to the surface following water permeation, causing variation of Bragg density gradient or crystallization gradient in the crystal. Hydrate precipitates when liquid constituent becomes oversaturated at the CA surface. Whereas if the hydration is controlled by the solid-state reaction mechanism, the distribution of the Bragg density or crystallization will not change since only water diffuses through the initial hydrate barrier.

Compared the Bragg density distribution of unhydrated CA crystal (Figure 3a) originated from sintering, with the crystal after 2 hours to 52 hours hydration, the higher Bragg density area (orange area) totally transformed into relatively low Bragg density area (yellow area) and the low Bragg density area (yellow area) was surrounded with the lower Bragg density areas (green and blue areas) enlarged towards the surface (Figure $3 \mathrm{~b}, 3 \mathrm{c}$ and $3 \mathrm{~d}$ ), which indicated the decrease of the degree of crystal ordering inside the crystal with the transfer of $\mathrm{CaO}$ and $\mathrm{Al}_{2} \mathrm{O}_{3}$ ions from the crystalline lattice. After 67 hours of hydration, the high Bragg density area (yellow area) totally transformed into low Bragg density area (blue area). From the SEM images of the hydrated CA for 3 days (Figure $\mathrm{Sib}$ ), there were homogeneously distributed nano-scale hydrogarnets on the surface of CA. It revealed that the hydration took place and hydrates precipitated on different sites within 3 days of hydration. Thus, the throughsolution reaction mechanism was confirmed.

Figure 4 shows the reconstructed ${ }_{3} \mathrm{D}$ images of the phase within the single CA crystal as a function of time during 3 days of hydration. It is coded in colour from dark blue to red (from -1.0o to 1.00 radians). All images are on the same scale, but the phase values are all relative within each image, and can only be compared within each image; there is an arbitrary offset between images. Positive value indicates tensile strain and negative value indicates compressive strain. The higher the value, the higher the strain is. There are three levels of strain in the crystal, positive, zero and negative. Positive and negative areas mixed up in the unhydrated CA 
crystal with transition zero value region, which we consider to represent the initial pattern of compressive and tensile strains originated from sintering (Figure 4a). Part of the initial pattern of strains observed in the crystal comes from interfacial mismatching effect between high Bragg density and low Bragg density parts, which remains throughout 3 days of hydration. The contact force between deposited hydrates and unhydrated crystals on the surface is considered to be directly proportional to the negative surface strain field. Although the strain effects are very subtle during 3 days of hydration, the negative area on the periphery of the cross section of the crystal enlarged at 3 days (Figure $4 \mathrm{e}$ ). Thus, the through-solution reaction mechanism was also confirmed by phase images.
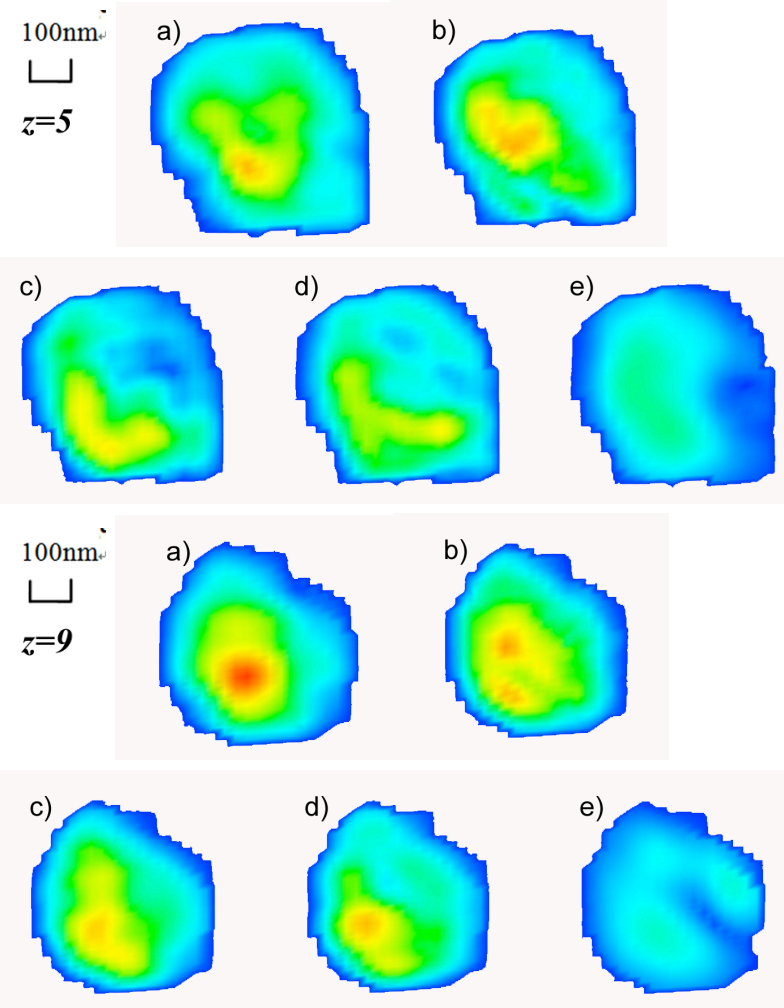

Bragg Diffraction Electron Density

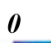

0.25

0.5

0.75

Figure 3. Cross sections through the reconstructed $3 \mathrm{D}$ images of the CA crystal showing the internal Bragg density variations during 3 days of hydration measured by BCDI. a) unhydrated CA. b) CA hydrated for $2 \mathrm{~h}$. c) CA hydrated for $24 \mathrm{~h}$. d) CA hydrated for $52 \mathrm{~h}$. e) CA hydrated for $67 \mathrm{~h}$.
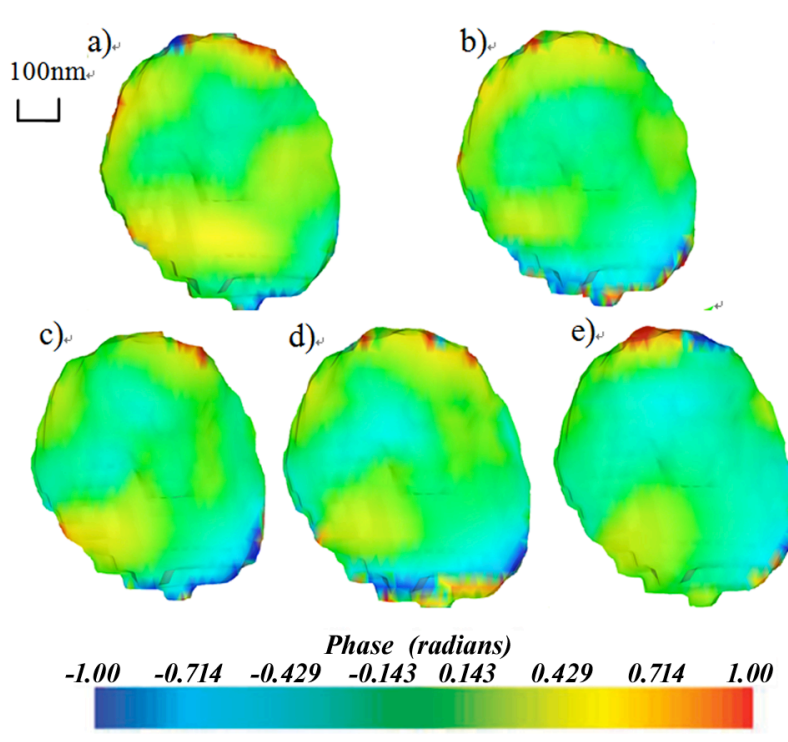

Figure 4. Corresponding maps showing the phase of the same cross sections as in Figure 3. a) unhydrated CA. b) CA hydrated for 2 h. c) CA hydrated for $24 \mathrm{~h}$. d) CA hydrated for 52h. e) CA hydrated for $67 \mathrm{~h}$.

According to defect-generated dissolution theories, the bulk dissolution rate of crystals is explained by the dissolution step waves originated from etch pits on the surface. ${ }^{27}$ Due to the strain field of a dislocation, the hollow core is opened up and an etch pit is formed for system far from equilibrium conditions, ${ }^{17,28}$ resulting in the consistent global dissolution throughout the surface of the crystal. ${ }^{27}$ Therefore the dissolution force of etch pit on the crystal surface is considered directly proportional to the positive surface strain field. However, there is no evidence for the surface strain field from etch pit during crystal dissolution. The possible reason is that the system is not far from equilibrium conditions.

From the analysis of the CA hydration measured by BCDI in this work, we can draw the following conclusions.

(1) Through-solution reaction (homogenized crystallization) mechanism during the hydration of CA was confirmed with BCDI, but defect-generated dissolution theory was not supported.

(2) Initial Bragg densities and strains in CA crystal were derived from sintering. The degree of crystal ordering decreased due to ion transfer during hydration. The strain coming from interfacial mismatch effect between high Bragg density and low Bragg density parts in the crystal remained throughout the experiment.

(3) The chemical phase transformation promoted the hydration process and the formation of new hydrates.

(4) The first change of Bragg density during hydration process was due to a big loss of Bragg density as seen in the image amplitude but not its phase.

\section{ASSOCIATED CONTENT}

\section{AUTHOR INFORMATION}




\section{Corresponding Author}

*lxp@tongji.edu.cn

*i.robinson@ucl.ac.uk

\section{Author Contributions}

\|These authors contributed equally.

\section{Notes}

The authors declare no competing financial interests.

\section{ACKNOWLEDGMENT}

We acknowledge the use of the Advanced Photon Source, which is operated by the U.S. Department of Energy under Contract No. DE-ACo2-o6CH11357. The BCDI instrumentation at the Advanced Photon Source beamline 34-ID-C was built with a National Science Foundation grant DMR9724294. The work was supported by National Natural Science Foundation of China (Project 51102181). The work at UMA was funded by MAT2010-16213 research grant (Spain) which is co-funded by FEDER. Ian Robinson is supported by EPSRC grant EP/Ioz2562/1 and a BBSRC Professorial Fellowship. Xianping Liu is supported by the State Scholarship Fund of China.

\section{Supporting Information}

$\mathrm{CaAl}_{2} \mathrm{O}_{4}$ synthetic procedure details. Basic characterization of $\mathrm{CaAl}_{2} \mathrm{O}_{4}$ by SEM (anhydrous and hydrated) and Rietveld quantitative phase analysis using laboratory X-ray powder diffractometer (anhydrous). This information is available free of charge via the Internet at http://pubs.acs.org.

\section{REFERENCES}

(1) Bensted, J. In Structure and Performances of Cements, 2nd ed.; Bensted, J., Barnes, P., Eds.; Spon Press: London, 2002; pp 114.

(2) Rivas Mercury, J. M.; De Aza, A. H.; Pena, P. J. Eur. Ceram. Soc. 2005, 25, 3269-3279.

(3) Goodwin, D. W.; Lindop, A. J. Acta Crystallogr., Sect. B 1970, 26, 1230-1235.

(4) Cottin, B. Cem. Concr. Res. 1971, 1, 273-284.

(5) Torréns-Martín, D.; Fernández-Carrasco, L.; Martínez-Ramírez, S. Cem. Concr. Res. 2013, 47, 43-50.

(6) Lamour, V. H. R.; Monteiro, P. J. M.; Scrivener, K. L.; Fryda, H. Microscopic studies of the early hydration of calcium aluminate cements. International Conference on Calcium Aluminate cement (CAC), Edinburgh, UK, 2001; 169-180.

(7) Scrivener, K. L.; Capmas, A. In Lea's Chemistry of Cement and Concrete, 4th ed.; Hewlett, P. C., Eds.; Arnold: London, 1998; pp 723727.

(8) Jensen, T. R.; Christensen, A. N.; Hanson, J. C. Cem. Concr. Res. 2005, 35, 2300-2309.

(9) Klaus, S. R.; Neubauer, J.; Goetz-Neunhoeffer, F. Cem. Concr. Res. 2013, 43, 63-69.

(10) Scrivener, K. L.; Cabiron, J. L.; Letourneux, R. Cem. Concr. Res. 1999, 29, 1215-1223.

(11) Rashid, S.; Turrillas X. Thermochim. Acta. 1997, 302, 25-34.

(12) Ge $\beta$ ner, W.; Trettin, R.; Rettel, A.; Müller, D. In Calcium Aluminate Cements; Mangabhai R. J., Eds.; E and FN Spon: London, 1990; pp 96.
(13) Bushnell-Watson, S. M.; Sharp, J. H. Cem. Concr. Res. 1990, 20, 677-686.

(14) Moehmel, S. Freiberg. Forschungsh. A 2003, 869, 1-238.

(15) Fujii, K.; Kondo, W.; Ueno, H. J. Am. Ceram. Soc. 1986, 69, 361364 .

(16) Robinson, I.; Harder, R. Nat. Mater. 2009, 8, 291-298.

(17) Frank, F. C. Acta Crystallogr. 1951, 4, 497-501.

(18) Lee, T. C.; Robertson, I. M.; Birnbaum H. K. Philos. Mag. A 199o, 62, 131-153.

(19) Chen, B.; Guizar-Sicairos, M.; Xiong, G.; Shemilt, L.; Diaz, A.; Nutter, J.; Burdet, N.; Huo, S.; Mancuso, J.; Monteith, A.; Vergeer, F.; Burgess, A.; Robinson, I. Sci. Rep. 2013, 3, 1177.

(20) Holler, M.; Diaz, A.; Guizar-Sicairos, M.; Karvinen, P.; Färm, E.; Härkönen, E.; Ritala, M.; Menzel, A.; Raabe, J.; Bunk, O. Sci. Rep. 2014, 4, 3857.

(21) Abbey, B. JOM. 2013, 65, 1183-1201.

(22) Pfeifer, M. A.; Williams, G. J.; Vartanyants, I. A.; Harder, R.; Robinson, I. K. Nature 2006, 442, 63-66.

(23) Aranda, M. A. G.; Berenguer, F.; Bean, R. J.; Shi, X. W.; Xiong, G.; Collins, S. P.; Nave, C.; Robinson, I. K. J. Synchrotron Radiat. 2010, 17, 751-76o.

(24) Harder, R.; Robinson, I. K. JOM. 2013, 65, 1202-1207.

(25) Fienup, J. R. Appl. Opt. 1982, 21, 2758-2769.

(26) Marchesini, S.; He, H.; Chapman, H.N.; Hau-Riege, S. P.; Noy, A.; Howells, M. R.; Weierstall, U.; Spence, J. C. H. Phys. Rev. B 20o3, 68, 140101.

(27) Lasaga, A. C.; Luttge, A. Science 2001, 291, 2400-2404.

(28) Cabrera, N.; Levine, M. M.; Plaskett, J. S. Phys.Rev. 1954, 96, 1153. 


\section{For Table of Contents Use Only}

In Situ Bragg Coherent Diffraction Imaging Study of a Cement Phase Microcrystal during Hydration

Xianping Liu, Miguel A.G. Aranda, Bo Chen, Peiming

Wang, Ross Harder, and lan Robinson

\section{TOC Graphic}

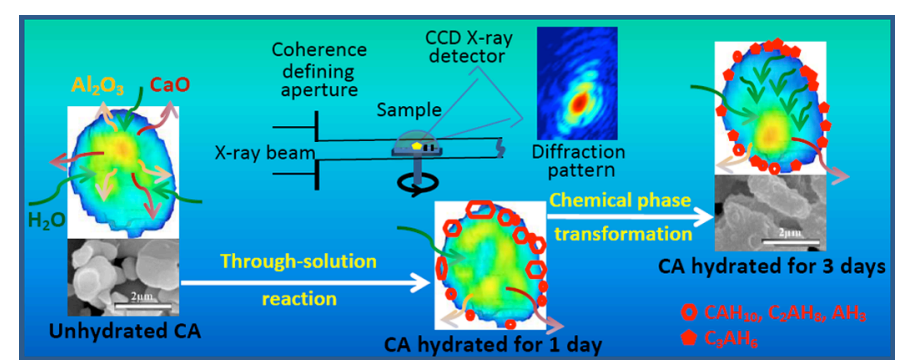

\section{Synopsis}

Results of Bragg coherent diffraction imaging (BCDI) confirm the ion migration and consumption during hydration of calcium monoaluminate (CA). The chemical phase transformation promotes the hydration process and the formation of new hydrates. There is a potential for the formation of hydrates near where the active ions accumulate. This work provides new evidence supporting the through-solution reaction mechanism of CA. 